\title{
Développement
}

\section{des larves de Dracunculus medinensis chez les Cyclops de l'Tle-de-France}

\author{
par Y.-J. GOLVAN et F.-A. LANCASTRE \\ (Laboratoire de Parasitologie, C.H.U. St-Antoine, 27, rue de Chaligny, 75 - Paris- $12^{\circ}$ )
}

\section{Résumé}

L'infestation des Cyclops de la région parisienne par les embryons de la Filaire de Médine est possible et donne des larves III infestantes pour l'homme.

Les embryons sont absorbés en une demi-heure et passent dans la cavité générale du Crustacé en 24 heures. Les larves II apparaissent au $8^{\circ}$ jour et les larves III vers le $15^{\circ}$ jour. Au-delà du $26^{\circ}$ jour, les Cyclops parasités commencent à mourir, la mort frappant d'abord les plus parasités. Par contre, la fécondité des Crustacés hébergeant des larves ne paraît pas affectée par la présence de ces parasites.

\section{Summary}

The infestation of Cyclops caught in different places around Paris is possible and produces III d. stage infective larvae. The embryos are swallowed by the crustacea after half an hour and passed trought the wall of the gut after the 24 th. hour. Second stage larvae appeared on the 8 th. day and III d. stage larvae on the 15 th. day. After the 26 th. day, the most heavily infected Cyclops began to die. It seems that the fecondity of the Crustaceans is not affected by parasitism. 
La Filaire de Médine (Dracunculus medinensis, Linné 1758) est un Nématode Dracunculidae qui détermine chez. les Mammifères, et singulièrement chez l'Homme, des accidents tégumentaires au terme de la migration des femelles parvenues à maturité. En France, nous observons maintenant cette parasitose avec une grande fréquence chez les Africains originaires du Mali et surtout de Mauritanie.

C'est l'explorateur de l'Asie centrale, Fedschenko (1871) qui découvrit le rôle d'hôte intermédiaire que joue le Crustacé Cyclops dans le cycle évolutif de ce parasite. Durant un demi-siècle nombre de travaux s'attachèrent à préciser le mode d'infestation du Crustacé et la transmission des larves infestantes qu'il contient aux hôtes définitifs soit naturels (Homme en particulier), soit expérimentaux (Chat). En ce qui concerne le mode de pénétration des larves du premier stade chez le Cyclops, les premiers Helminthologistes peuvent se répartir en deux écoles :

- l'une affirmait que les larves libérées dans l'eau au moment de l'immersion de la plaie cutanée pénétraient de façon active à travers la carapace chitineuse de l'Arthropode. Fedschenko lui-même, Blanchard (1890), Manson (1905) et Wenyon (1908) sont les représentants les plus célèbres de cette opinion. Pour eux, les embryons qui, par hasard, pouvaient être ingérés par le Cvclops étaient détruits par ses sucs digestifs. Ils considéraient comme la preuve de ce mécanisme, la présence des larves de la Filaire dans la cavité généra!e du Crustacé très peu de temps après la mise en présence de l'un et l'autre protagonistes. Ce délai bref impliquait un passage par voie transtégumentaire ;

- l'autre pensait que l'infestation des Cyclops se faisait par ingestion des larves et qu'elles passaient ensuite par effraction de la muqueuse digestive dans la cavité générale. Cette théorie est défendue notamment par Leiper (1907), Roubaud (1913), Chatton (1918), Faviley, et Liston (1924), Manson-Bahr (1936), Moorthy (1937 et 1938), Onabamiro (1950) et Muller (1966). On sait que c'est effectivement par cette voie et cette voie seulement que se fait la pénétration des larves de la Filaire de Médine dans le corps des Cyclops. Il y a donc sur ce point convergence entre le développement de Dracunculus medinensis et celui des vraies Filaires, en particulier celles de l'Homme, qu'elles aient des adultes dans le tissu cellulaire sous-cutané, dans les vaisseaux lymphatiques ou le péritoine.

Ajoutons que quelques auteurs, tels Sambon (1905), croyaient à la nécessité d'un second hôte intermédiaire alors qu'à l'inverse, d'autres pensaient que le cycle était direct sans qu'il soit besoin du passage par l'organisme d'un Invertébré [Plehn, Busk cités par Leiper (1907)], soit par ingestion des embryons par l'hôte définitif, soit par pénétration active des larves à travers la peau de ce dernier.

Mis à part le travail de Camille Desportes (1938) qui étudia le développement d'un Dracunculidae, parasite des Couleuvres, aucun auteur de langue française n'a récemment publié sur ce sujet. Ceci est d'autant plus incompréhensible que cette parasitose sévit avec prédilection dans les pays de l'Afrique de l'Ouest (Mauritanie, Mali, Sénégal, Guinée) qui ont gardé avec l'ancienne métropole des liens économiques et culturels privilégiés. 
Il faut dire que la Dracunculose n'a rien d'une maladie spectaculaire et qu'elle est généralement considérée comme bénigne. Ceci serait probablement à revoir en raison des nombreux accidents articulaires ou toxiques que nous pouvons tous les jours constater chez les sujets hospitalisés porteurs d'une Filaire de Médine.

Tous les travaux sur le développement de $D$. medinensis ont été réalisés dans les pays où sévit cette parasitose (Turkestan soviétique, Afrique occidentale francophone et anglophone, Inde) Il faut cependant signaler une exception, le travail de Manson (1893) qui réussit le cycle en travaillant avec des Cyclops des iles britanniques. A notre connaissance, aucune étude n'a été faite sur l'infestation possible des Cyclops de France.

Le but de notre expérimentation n'a pas été de donner une nouvelle description des stades larvaires qui furent bien observés, en particulier par Roubaud (1913) et Moorthy (1938), mais de vérifier la possibilité d'obtenir des larves infestantes de D. medinensis chez les Cyclops récoltés dans la région parisienne. Bien entendu, l'importance épidémiologique d'une telle constatation est purement théorique puisque les sujets porteurs de filaire de Médine n'ont pas pour habitude de faire leurs ablutions dans les «lacs» du Bois de Boulogne et que les Parisiens ne s'y abreuvent pas.

\section{Matériel et Méthodes.}

\section{$1^{\circ}$ Les Embryons DE Dracunculus medinensis.}

Nous les avons facilement récoltés chez plusieurs malades porteurs de plaies cutanées au niveau de la partie inférieure de la jambe. Il s'agissait toujours de travailleurs originaires de Mauritanie. L'émission des embryons était provoquée en aspergeant le pourtour de la plaie avec un jet de chlorure d'éthyle. Après quelques secondes sourd un liquide opalescent qui est récolté à la pipette. Il contient une multitude de larves du premier stade, à mouvements assez lents, mais qui s'accélèrent dès qu'on les place dans l'eau pure. Environ deux heures après l'émission, nous répartissons ces larves dans des récipients de verre (ventouses ou petits bocaux) d'environ $50 \mathrm{ml}$. de capacité. Chaque récipient contient au moins 100 larves I. Elles y survivent plus de trois jours à la température du laboratoire $\left(22\right.$ à $\left.23^{\circ} \mathrm{C}\right)$ et c'est cette température qui sera maintenue durant toute la durée de l'expérimentation.

\section{$2^{\circ}$ Les Cyclops, sp.}

Ils proviennent d'un élevage maintenu au laboratoire depuis plus d'un an. Ils se reproduisent abondamment dans l'eau du robinet \& vieillie 》 (c'est-à-dire conservée à l'abri des poussières pendant un mois durant lequel elle se débarasse de ses produits chlorés) qui sert à alimenter nos aquaziums d'élevages de Planorbes. Les premiers individus de cette souche de Cyclops proviennent des étangs de Hollande, dans la forêt de Rambouillet (département des Yvelines). 
$3^{\circ}$ Mode d'infestation des Cyclops.

A. - Une trentaine de Cyclops sont répartis, à l'aide d'une pipette Pasteur à large ouverture, dans chacun des pots de $50 \mathrm{ml}$ contenant déjà les larves I de la Filaire. Nous avons également tenté l'infestation dans des bocaux de verre de 5 litres, mais nous avons constaté que, dans ce cas, la survie des Cyclops parasités est bien plus précaire et qu'ils disparaissent, à infestation égale, bien plus rapidement que dans les pots de $50 \mathrm{ml}$. Ce fait a déjà été constaté par Moorthy (1938) et il serait en rapport avec une insuffisance de l'oxygénation. L'eau près du fond de ces grands récipients ne contiendrait pas assez d' $\mathrm{O}^{2}$ dissout et obligerait les Crustacés parasités à nager sans cesse pour se maintenir près de la surface, ce qui les épuiserait rapidement.

B. - Pendant les cinq premières heures de la mise en contact, toutes les demiheures, les Cyclops sont examinés à la loupe binoculaire. Par la suite, et pendant 25 jours, il n'y aura plus qu'un examen quotidien. Au bout d'une demi-heure, de nombreux Cyclops ont déjà des larves dans ieur estomac. Ces larves sont parfaitement mobiles et paraissent tout à fait intactes. A la fin des cinq premières heures pratiquement toutes les larves avaient été ingérées et certains Cyclops en hébergeaient de deux à cinq dans leur tube digestif. Etant donné l'extrême mobilité des larves I dans l'eau (ce qui rend leur observation continue presque impossible), il faut admettre de la part des Cyclops, une chasse active. Elles doivent être considérées comme des proies par le Crustacé.

C. - Au bout de vingt-quatre heures, les larves I sont dans la cavité générale. Cette traversée de la paroi digestive paraît s'opérer sans conséquence fâcheuse pour le Cyclops. Par fa suite, alors que nos Cyclops infestés vieillissaient, nous avons observé que nous avions bien plus de femelles infestées que de mâles. Ceci est peut-être dû à une plus grande résistance de ce sexe. Ces femelles paraissaient parfaitement normales sauf, peut-être un peu moins agiles que les femelles non parasitées. Elles portaient leurs habituelles grappes jumelles d'œufs accrochées aux derniers segments abdominaux. Ces œufs étaient parfaitement fertiles et donnaient naissance à de nombreux petits Cyclops. Tous les jours, des Cyclops parasités ont été prélevés, écrasés entre lame et lamelle. Les larves I libérées sont bien reconnaissables à leur long appendice caudal effilé. Elles sont toujours capables de résister plusieurs heures dans l'eau pure.

D. - Les premières larves du second stade apparurent au bout de huit jours, d'abord en petit nombre, et surtout chez les Cyclops hébergeant plusieurs larves. Puis, le nombre de ces larves II augmente jusqu'aux alentours du quinzième jour, date à laquelle, ces larves sont largement majoritaires. Plusieurs observations doivent être faites.

a) Chez tous les Cyclops n'hébergeant qu'une seule larve, la transformation des larves I en larves II n'est pas synchrone, et se fait plus vite chez certains que chez d'autres appartenant au même bocal.

b) Chez les Cyclops hébergeant plusieurs larves, la vitesse d'apparition des larves II est inversement proportionnelle à la quantité de larves ingérées. Chez quelques 
Cyclops qui avaient ingéré cinq ou six larves du premier stade, nous avons même pu constater la présence simultanée de larves des stades I, II et III.

E. - Le comportement des larves II extraites des Cyclops est différent de celui des larves I. Elles sont bien moins mobiles, ne se déplacent plus rapidement dans l'eau, mais rampent sur le fond du récipient. De plus, elles éclatent rapidement dans l'eau pure, évaginant la totalité de leur œsophage. Elles ne peuvent être étudiées et maintenues en vie que dans une solution tiède de $\mathrm{CINa}$ à $6 \%$. Tout comme les larves II, les larves III éclatent dès qu'on les place dans l'eau pure. Ce stade III est le stade infestant.

F. - Au vingt-deuxième jour, de l'infestation, presque toutes les larves sont au stade III. Théoriquement, les Cyclops sont capables d'infester l'Homme à cette date. Malheureusement, à partir de ce moment, le nombre des Cyclops infestés diminue dramatiquement et, au vingt-sixième jour, nous n'avions pratiquement plus de Cyclops infestés à notre disposition.

Nous avons infesté un jeune chat qui est actuellement conservé en observation au laboratoire.

La disparition des Cyclops très infestés après le vingt-sixième jour, paraît être un phénomène pathologique. Moorthy en 1930 avait observé la même mortalité intense chez les Cyclops polyparasités (certains contenaient jusqu'à 20 larves !). Dans le milieu naturel, il n'a jamais trouvé que des Cyclops contenant une seule larve. L'infestation expérimentale, parce qu'elle est faite en vase clos aboutit à des infestations massives qui sont incompatibles avec la survie prolongée des hôtes intermédiaires.

\section{Bibliographie}

Blanchard (R.), 1890. - Les Animaux parasites introduits par l'eau dans l'organisme. Revue d'Hygiène, XII (9-10).

Chatron (E.), 1918. - Observations sur le Ver de Guinée. Preuve expérimentale de l'infestation des Cyclops par voie digestive. Bull. Soc. Path. Exot., XI, 338-348.

Desportes (C.), 1938. - Filaria asophogea Polonio 1859, parasite de la Couleuvre d'Italie est un Dracunculus très voisin de la Filaire de Médine. Ann. Parasit. Hum. Comp., XVI, 305-326.

FAIRLEY (N. H.) et Liston (W. G.), 1924. - Studies in the transmission of Dracunculus medinensis. Ind. J. Med. Res., XII, 93-103.

FEDsChenKo (B. A.), 1871. - Formation et croissance de Filaria medinensis L. (en russe). Izv. Imp. Obsh. Limb. Estet. Antrop. Moskva, VIII (1), 71-78.

LeIPER (R. T.), 1907. - The etiology and prophylaxis of Dracontiasis. Brit. Med. J. (19 janvier), 129-132.

Manson (P.), 1893. - «Guinea Worm » in Davidsons Hygiene and Diseases of Warm Climates », 947-961.

Manson (P.), 1905. - Lectures on tropical diseases for 1905. Constable, London. 
Manson-Bahr (P. E. C.), 1944. - Synopsis of Tropical Medicine, 1 vol., 204 p. Cassell and Co, London.

Moorthy (V. N.), 1937. - A redescription of Dracunculus medinensis. J. Parasitology, XXIII, 220-224.

MOORTHY (V.N.), 1938. - Observations on the development of Dracunculus medinensis larvae in Cyclops. Am. J. Hyg., XXVII (2), 437-460.

MULLeR (R. L.), 1966. - First step in the laboratory maintenance of Dracunculus medinensis. Trans. Roy. Soc. Trop. Med. Hyg., LX (4), 431-432.

Onabamiro (S. D.), 1950. - A technique for studying infection of Dracunculus in Cyclops. Nature, $C L X V, \mathrm{n}^{\circ} 4184,31$.

Roubaud (E.), 1913. - Observations sur la biologie du Ver de Guinée. Infection intestinale des Cyclops. Bull. Soc. Path. Exot., VI, 281-288.

SAmbon (L. W.), 1905. - Discussion on Guinea Worm, British Medical Association.

Wenyon (C. M.), 1908. - III rd Rep. Wellcome Res. Lab. Khartoum., 132-135. 